\title{
Cuidados y derechos El avance hacia la democratización de los cuidados
}

\section{(4) Dolors Comas-d'Argemir}

Universidad Rovira i Virgili, Tarragona, España

ORCID: https://orcid.org/oooo-0002-0385-0436

Correo electrónico: dolors.comasdargemir@urv.cat

\section{Resumen}

El artículo plantea que el cuidado reviste un interés particular para la antropología económica, ya que incorpora tendencias aparentemente contradictorias: permanecer en el ámbito familiar, fuera de las relaciones de trabajo/capital, y por otro lado, hallarse en un proceso de mercantilización y de construirse como una cuestión social y política. Ambas tendencias se sitúan a su vez en la tensión entre los mecanismos de producción y de reproducción social. El artículo aborda el tema del cuidado como derecho y, por tanto, como una cuestión de alcance político. Partiendo de la constatación de que el cuidado recae de forma desproporcionada en las mujeres y en las familias, sugiere la necesidad de su redistribución equitativa entre familia, Estado y comunidad, y entre mujeres y hombres, para avanzar hacia su democratización.

\section{Social care and rights. Moving towards the democratization of social care}

\begin{abstract}
The article argues that care is of particular interest to economic anthropology, as it incorporates seemingly contradictory tendencies: to remain within the family sphere, outside of labor/capital relations and, on the other hand, to be in the process of commodification and to be constructed as a social and political question. Both tendencies are in turn located in the tension between the mechanisms of production and social reproduction. The article deals with the issue of care as a right and, therefore, as an issue of political scope. Starting from the observation that care falls disproportionately on women and families, it suggests the need for its equitable redistribution between family, state and community, and between women and men, in order to advance towards its democratization.
\end{abstract}

El texto de este artículo es fruto de una conferencia impartida en Buenos Aires el 18 de septiembre de 2017, en la Jornada Internacional “Derechos y políticas de cuidado y apoyo", organizada por AMIA (Asociación Mutual Israelita Argentina) y por el Consejo Nacional de Coordinación de Políticas Sociales de la Presidencia de la Nación. Va precedido de una introducción de carácter académico que sitúa la importancia de analizar los cuidados en el marco de la antropología económica.

\section{Palabras clave}

Cuidados; Reproducción Social; Género; Justicia Social; Derechos Ciudadanos

\section{Key words}

Care; Social Reproduction; Gender; Social Justice; Citizen Rights 


\section{Cuidados e direitos. Avançando para una democratização do cuidado}

Palavras-chave

Cuidado; Reprodução Social; Gênero; Justiça Social; Direitos do Cidadão

\section{Resumo}

O artigo argumenta que o cuidado é de particular interesse para a antropologia econômica, uma vez que incorpora tendências aparentemente contraditórias: permanecer na esfera familiar, fora das relações trabalho/capital e, por outro lado, encontrar-se num processo de mercantilização e ser construído como uma questão social e política. Ambas tendências localizam-se, por sua vez, na tensão entre os mecanismos de produção e da reprodução social. $\mathrm{O}$ artigo trata da questão do cuidado como um direito e, portanto, como uma questão do âmbito político. Partindo da constatação de que o cuidado recai desproporcionalmente sobre mulheres e famílias, sugere a necessidade de sua redistribuição equitativa entre família, Estado e comunidade e entre mulheres e homens, a fim de avançar para uma democratização.

\section{El cuidado en el marco de la antropología económica}

Tratar el tema de los cuidados es hacer referencia al trabajo reproductivo, esencial para la creación y mantenimiento de las personas, de las familias, de las sociedades. Buena parte del cuidado se realiza fuera de las relaciones trabajo/capital en el ámbito familiar y en el comunitario, aunque hoy también intervienen otros agentes, como son los servicios suministrados desde la esfera pública y desde el mercado. Cuidar es alimentar, proporcionar vivienda y vestido, criar a niños y niñas, asistir en la enfermedad, dar consejos, ayuda práctica y emocional. Nos cuidamos a nosotros mismos y también a las personas de nuestro entorno inmediato. Se trata de actividades diversas y desiguales que pueden hacerse de forma continuada o esporádica según el ciclo vital de las personas o de coyunturas críticas. Las mujeres son quienes cuidan mayoritariamente: a sus propios hijos y a los de otra gente, así como a familiares enfermos o en situación de dependencia. Y ellas son mayoritariamente las contratadas para cuidar en empresas privadas, en servicios públicos y en hogares. El cuidado de hijos y familiares no está remunerado; cuando el trabajo de cuidados se paga, la remuneración es baja o muy baja.

Desde el punto de vista de la antropología económica, el análisis de los cuidados reviste un interés particular por incorporar tendencias aparentemente contradictorias. Los trabajos de cuidados persisten fuera de la lógica mercantil y al mismo tiempo se mercantilizan; se asocian a la esfera familiar y privada, y al mismo tiempo se socializan y politizan. Las tensiones entre estas tendencias opuestas constituyen un interesante marco de análisis.

La primera tendencia (estar fuera de las relaciones trabajo/capital) parece contradecirse con lo que ha sido uno de los elementos definidores del sistema capitalista: la mercantilización de todas las cosas (Wallerstein, 1988), también del trabajo humano. Tenía razón Karl Polanyi (1989) cuando afirmaba que el trabajo (así como la tierra o el dinero) es una "mercancía ficticia" ya que no es producido de forma finalista para su venta, y por ello la sociedad tuvo que arbitrar mecanismos institucionales (regulaciones laborales, convenios, mínimos salariales) para evitar que el trabajo se viera sometido a la única ley del mercado, puesto que esto hubiera llevado a una explotación creciente de las personas y a su aniquilación. Pero no todo el trabajo se mercantilizó, como es 
el caso de las actividades que se dirigen a la propia subsistencia, las que contribuyen a la reproducción humana y el voluntariado. Preguntarse pues por qué el trabajo se mercantiliza implica también preguntarse por qué una parte de él no lo hace (Comas d'Argemir, 1998).

Se ha podido mostrar que la lógica mercantil no ha penetrado de forma homogénea en todas las instancias de la vida y la sociedad, y que las transiciones al capitalismo son incompletas, inacabadas y con múltiples facetas. Más aún, los individuos, los hogares o las comunidades pueden realizar actividades inscritas en relaciones de producción de distinta naturaleza: es la "pluralidad de bases económicas", en términos de Maurice Godelier (2017). Así sucede, por ejemplo, en las unidades domésticas que son células de la economía popular (Coraggio, 1999) o de la economía social y solidaria (Presta y Landaburu, 2014), donde se muestra la escisión entre la racionalidad económica orientada a obtener ganancias en la lógica capitalista de producción, circulación y consumo de bienes, y la orientada a la reproducción de la vida en el ámbito familiar y comunitario.

La familia es la institución que se considera responsable del cuidado de sus miembros. Desde la antropología se ha analizado cómo el género y el parentesco constituyen las relaciones que se activan para proporcionar los cuidados en el marco familiar (Carsten, 2004; Drotbohm y Alber, 2015; Comas d'Argemir y Soronellas, 2019). Las normas relacionadas con el don, la reciprocidad y la moralidad atraviesan estas obligaciones, y hemos mostrado que el género impregna a su vez los propios principios morales y éticos (Comas d'Argemir, 2017). Es así que el cuidado ha sido calificado como un "trabajo por amor" (Finch y Groves, 1983) y se han destacado también sus dimensiones éticas (Gilligan, 2013).

Se haga por amor o por obligación moral, lo cierto es que el cuidado no pagado que se realiza en la familia tiene valor económico, aunque no sea visible. Por esto situamos los cuidados en lo que denominamos "economía del afecto", en un doble sentido: porque este trabajo no pagado tiene valor económico (lo que queda de manifiesto cuando se efectúa en el marco del mercado o del Estado), y también porque "economiza" gasto público, al privatizar la responsabilidad de los cuidados en las familias. La economía feminista ha podido demostrar el valor económico del trabajo que se realiza en los hogares y el impacto de la producción doméstica sobre las economías nacionales. Ha cuestionado que el mercado sea el único estándar de valor y ha llamado la atención respecto de la importancia del trabajo no pagado, las actividades de aprovisionamiento y mantenimiento, los procesos de socialización y la transmisión del conocimiento cultural (Harris y Young, 1981; Katz, 2001; Carrasco, Borderías y Torns, 2011).

La tendencia opuesta a esta permanencia de los cuidados en el ámbito familiar y comunitario es su externalización hacia otros agentes. El incremento de las necesidades de cuidado y los cambios en las estructuras familiares han contribuido a la externalización de los cuidados hacia los servicios públicos, los servicios mercantiles y las empleadas domésticas. Los cuidados constituyen un sector ocupacional en expansión que realizan mayoritariamente mujeres migrantes, en un contexto económico neoliberal y de migraciones internacionales.

Pagar para que alguien realice los trabajos domésticos y de cuidados no es algo nuevo. Sirvientas, nodrizas, criadas, empleadas domésticas - a menudo procedentes de contextos rurales - han participado en el trabajo reproductivo de forma remunerada a lo largo de la historia (Wikander, 2016; Offenhenden, 2017). A lo largo del siglo XX se produjo en Europa un declive de este tipo de trabajo debido a las nuevas oportunidades de empleo que se ofrecía a las jóvenes que habían accedido a la educación y a la expansión de la figura del ama de casa. Mientras que el mercado proporcionaba los bienes 
que se necesitaban en la vida cotidiana (como alimentos, vestido o casa), los servicios reproductivos, incluyendo el cuidado, eran proporcionados por las mujeres de forma no pagada en la familia o en el contexto comunitario. Eventualmente se contrataban empleadas por horas y solo los más ricos tenían empleadas permanentes (criadas). El empleo doméstico, pues, no es nuevo. Lo que sí es una novedad es su magnitud, su mayor vínculo con los cuidados y su internacionalización, y conforman un nuevo orden doméstico mundial (Ehrenreich y Hochschild, 2003).

Hay que destacar que en los últimos dos decenios se ha incrementado exponencialmente el recurso al trabajo de cuidados pagado, tanto por el contrato directo de las familias como por la expansión de los servicios de cuidado públicos y privados. Los trabajos remunerados de cuidados se caracterizan por tener salarios bajos, estar poco valorados y tener dificultades de profesionalización (Razavi y Staab, 2010). Son labores precarizadas, generizadas y racializadas, que realizan mayoritariamente mujeres migrantes. Más recientemente, empresas mercantiles, a veces conformadas por grandes corporaciones, han entrado también en el sector de los cuidados. Es la mercantilización de la intimidad (Constable, 2009; Boris y Parreñas, 2010).

Estas dos tendencias contradictorias (la persistencia de la no mercantilización del trabajo doméstico y de cuidados y la mercantilización de una parte de él) se sitúan en otro eje de tensión, el que se produce entre la acumulación de capital y la reproducción social. Hay que recordar que la producción de bienes y servicios y la reproducción social son parte de un proceso integrado: no hay sistema productivo ni sociedad alguna que puedan existir sin que se reproduzca la vida y se sostenga; no hay producción sin reproducción.

La reproducción social se asume por medio de cuatro tipos de relaciones e instituciones: 1) la familia, mediante un trabajo que no es remunerado; 2) el Estado, mediante servicios y prestaciones que constituyen una especie de salario social; 3) el mercado, que proporciona servicios con fines lucrativos; y 4) la comunidad en sus múltiples formas (redes familiares o vecinales, entidades sin fines lucrativos). Así sucede con el cuidado, que abarca todas estas dimensiones y constituye en sí mismo un sistema de organización social (Thelen, 2015). Es interesante ver, por ejemplo, cómo esto se materializa en el cuidado infantil en el caso de Argentina en el estudio llevado a cabo por Valeria Esquivel, Eleonor Faur y Elizabeth Jelín (2012). El predominio de cada uno de estos agentes en la provisión de cuidados tiene que ver con la tensión entre las lógicas de acumulación de capital y las lógicas de reproducción social, lo que se expresa en la fortaleza o debilidad de las políticas públicas en cada país; y es resultado de la negociación (o conflicto) constante entre quienes tienen el poder económico y político y quienes demandan derechos sociales. Tal como señala Tithi Bhattacharya (2013), esta dependencia contradictoria entre producción y reproducción es esencial para entender la economía política de las relaciones de género.

Mientras las necesidades de cuidados se han ido incrementando (como resultado del envejecimiento y la mayor supervivencia de las personas con enfermedades crónicas y discapacitadas), la capacidad de cuidado de las familias ha disminuido, debido a los propios cambios familiares y a la menor disponibilidad de las mujeres. Es así como los cuidados adquieren una naturaleza social y política y son un verdadero desafío para el siglo XXI (Esquivel, 2011; Tobío, Agulló, Gómez y Martín, 2011). Y con ello surgen múltiples preguntas: ¿ es un derecho ser cuidado?; ¿lo ha de ser de forma equivalente al derecho a la educación o a la sanidad?; ¿en qué condiciones? Sabemos que el cuidado está suministrado desproporcionadamente por las mujeres y por la familia, entonces, ¿cómo hay que redistribuirlo? ¿En qué grado se han de involucrar la familia, los poderes públicos o la comunidad? ¿Se han de involucrar los hombres? ¿Es un derecho cuidar? En definitiva, ¿qué elementos han de existir para conseguir una democratización de 
los cuidados? Este es el tema que abordaremos a lo largo de este texto, que es de un carácter marcadamente propositivo en la medida en que defiende la necesidad de democratizar los cuidados.

\section{Cuidados y derechos}

El cuarto pilar del sistema de bienestar. Así se ha calificado el derecho a ser cuidado y a cuidar: una nueva generación de derechos que viene a engrosar los derechos sociales conseguidos en siglos anteriores (el derecho a la educación, a las pensiones, a la sanidad). El derecho a ser cuidado y el derecho a cuidar forman actualmente parte de debates sociales y políticos en distintos países. El cuidado desborda así el marco familiar, donde se ha encuadrado tradicionalmente, para pasar a ser un asunto social y político. El cuidado ha sido, es, cosa de mujeres, y actualmente entendemos que los hombres se han de incorporar también, no solo como una cuestión de justicia de género - que sí lo es-, sino también porque son imprescindibles para atender las crecientes necesidades de cuidados. El cuidado está desproporcionadamente provisto por la familia de forma no pagada, pero es hoy también un ámbito ocupacional en expansión. Pagamos para que alguien cuide (en nuestras casas, en servicios públicos o privados) y este alguien (mujeres, generalmente) puede proceder de lugares lejanos, de países lejanos. Las necesidades de cuidado, algo tan íntimo, cargado de emociones, de tensiones y conflictos, de ética y de moralidad, también de materialidad, forman parte hoy de un escenario internacional, globalizado.

Es en este escenario complejo donde se sitúa mi reflexión. Con una propuesta articuladora: para poder ejercer el derecho a ser cuidado y a cuidar hay que democratizar los cuidados. Este es mi mensaje: vincular derechos y cuidados a la justicia social y a la equidad de género.

Pero vayamos por partes, para profundizar un poco más en el porqué del interés social actual por los cuidados, para explorar de dónde venimos y qué nos condiciona, y para comentar también qué podemos hacer. Y partiremos de algunas constataciones, que nos permiten afrontar el cuidado desde diversos ángulos y en su complejidad.

Estamos, a nivel mundial, en un momento de transición demográfica: hemos pasado de un régimen de alta mortalidad y alta natalidad, a otro en que se invierten las variables anteriores: reducción de la mortalidad y disminución de la natalidad. Esta dinámica conforma el envejecimiento de nuestras sociedades: vivimos más años y hacemos menos hijos/as. Resultado: nuestras sociedades envejecen. Dos son las razones más comúnmente atribuidas a este proceso:

El descenso de la fecundidad, que ha tenido un insospechado decrecimiento desde la década del sesenta, de manera que ya en los años ochenta las tasas de fecundidad de muchos países europeos y de EEUU se situaron por debajo de las tasas de reemplazo, como hoy sucede también en Chile, Brasil, China, Japón o Rusia, tal como muestra la Figura 1. He de señalar que España tiene una de las tasas de fecundidad más bajas del mundo ( 1,3 hijos por mujer). Solo los países africanos están experimentando actualmente un crecimiento demográfico.

La drástica reducción de la mortalidad en todas las etapas de la vida (Figura 2). Desde los años setenta esta reducción es especialmente importante entre las personas de más de 55 años, lo que comporta una mayor proporción de los adultos mayores de más de 80 años y un notable incremento de la esperanza de vida. Los datos son espectaculares. 
Figura 1. Tasas de fecundidad

Figura 2. Esperanza de vida al nacer


Es el triunfo de la longevidad, que ha de ser percibido como logro del progreso social y de la democratización de la supervivencia y no como una catástrofe (Comas d’Argemir y Roigè, 2018). Y desde este foco hay que afrontar un futuro no demasiado lejano (si ninguna contrariedad modifica las previsiones) en que el número de personas adultas y adultas-mayores será mucho mayor que el número de jóvenes, algo que las migraciones o un incremento de la natalidad pueden mitigar, pero no frenar. $\mathrm{Y}$ esto tiene consecuencias para las necesidades de cuidados.

Tenemos, por un lado, una infancia que disminuye en número, aunque al mismo tiempo, se incrementan las exigencias en su cuidado (alimentación, salud, educación, socialización, atención directa). Además, hay también las necesidades de cuidado de la denominada "vejez-frágil", es decir los adultos-mayores que no pueden valerse por sí mismos. Finalmente, deben añadirse las situaciones de discapacidad o de limitación derivadas de las enfermedades crónicas, alteraciones congénitas, así como de la siniestralidad vial y laboral. Son realidades que conectamos a través de lo que llamamos 
cuidados, pero cada una de las cuales tiene su especificidad, y dentro de cada una hay, además, singularidades.

Dos aspectos a resaltar en este escenario de incremento en las necesidades de cuidado y apoyo, ya apuntando propuestas.

Las políticas sociales son esenciales en la construcción de un sistema de cuidados sostenible. Proporcionar servicios de cuidado (para la infancia, los adultos mayores, las personas con discapacidades) no solo alivia a las familias (y sobre todo a las mujeres) de su labor de cuidar, sino que son asequibles para toda la población, incluyendo a los más pobres. Una apuesta por una red de empresas públicas, o de colaboración públicoprivadas (no lucrativas) equivalente a las redes educativas o sanitarias es una opción viable, y es social y económicamente interesante.

Por otro lado, el cuidado es un sector generador de empleo. Los empleos de cuidados aumentarán en los próximos años, tanto en el sector público como en el privado. Apostar por el desarrollo profesionalizado de los empleos de cuidados, con condiciones laborales y salarios dignos, es abordar las necesidades presentes y de futuro en términos realistas, teniendo en cuenta la evolución demográfica de nuestras sociedades. Es un sector muy intensivo en lugares de trabajo y en el cual hay mucho margen de crecimiento; es un trabajo que no se puede deslocalizar; combate la economía sumergida y, por tanto, posibilitaría obtener más impuestos; vincularía a millares de mujeres a los derechos sociales; haría más atractivo para los hombres obtener este tipo de empleos; implicaría el derecho a ser cuidado como obligación colectiva.

¿En qué situación estamos hoy? ¿De dónde partimos? ¿Es satisfactorio el sistema de cuidados? No, no lo es, porque actualmente se asienta en injusticias sociales y en injusticias de género que han provocado una crisis profunda y la necesidad de reformar el sistema desde una lógica transformadora.

\section{La crisis de los cuidados y las injusticias sociales}

El incremento de las necesidades de cuidados se produce en un contexto de profundo cambio en las familias y en la vida de las mujeres. Las familias, cada vez más reducidas, y con mujeres muy ocupadas, no pueden absorber las necesidades de cuidados, cada vez mayores. La falta de implicación de los hombres en los cuidados, la fragmentación de las redes de apoyo y comunitarias, la falta de servicios de esta categoría contribuyen también a engrosar el problema. Es lo que se ha denominado "crisis de los cuidados", que se ha expresado con fuerza en los países europeos inicialmente, pero que es actualmente un fenómeno generalizado que se manifiesta en particular en los ámbitos urbanos.

Sabemos que hay una injusticia de género vinculada al papel de las mujeres en los cuidados. Las necesidades de cuidados son universales y alguien los ha de asumir, no hay elección. El hecho de que el cuidado recaiga principalmente en las mujeres tiene repercusiones en las trayectorias laborales y sociales a lo largo de sus vidas. El cuidado es un gran devorador de tiempo, que no se puede destinar a otras cosas y limita las oportunidades. Y esto implica salarios más bajos, pensiones más bajas, pobreza femenina. También los hombres cuidan de su familia, pero de una manera diferente. En nuestro contexto cultural, la obligación primaria de los hombres es aportar los recursos materiales para vivir, y esto es una prescripción gravosa de cumplir que también implica poner en juego capacidades y tiempo. Pero esto sí se contabiliza y se remunera. La cuestión está, pues, en que la división sexual del trabajo sitúa a hombres y mujeres en una relación desigual. Los hombres apenas se han implicado en el cuidado directo, aunque hay variaciones según países, edades y formas de socialización. Se trata en 
todo caso de un tema no resuelto en términos de igualdad de oportunidades entre hombres y mujeres; y es en este punto donde se encuentra un conflicto subyacente, no siempre visible, no siempre explícito, pues se asienta en unos patrones de género y en una estructura social informal que tiene efectos discriminatorios, pues consume las energías, oportunidades y capacidades de las mujeres y limita en la práctica el ejercicio de la ciudadanía.

Sabemos que hay también una injusticia de clase asociada a los cuidados, con múltiples facetas. Las desigualdades sociales inciden en las desigualdades de salud, y los sectores populares más desfavorecidos envejecen en peores condiciones y viven menos años. Los hogares pobres, por otro lado, suelen tener más hijos a su cargo, y más dificultades para criarlos. De hecho, los sectores pobres acumulan una doble crisis de cuidados: tienen mayores necesidades de ellos (tanto de infantes como de adultos mayores, lo que supone más presión sobre las mujeres) y las mujeres de este estrato social tienen más dificultades para mantener su empleo.

Y esta injusticia social se proyecta también en las trabajadoras del cuidado. Cuando los sistemas públicos de cuidados son débiles y las necesidades son elevadas, los hogares de clase media y alta solucionan el problema contratando cuidadoras domésticas, $o$ utilizando servicios del mercado. Esto no es nuevo; hace muchos años ya que mujeres de zonas rurales o de sectores populares urbanos vienen trabajando como niñeras, empleadas domésticas y cuidadoras de adultos mayores. Lo que es nuevo es el carácter internacional de las migraciones para cuidar. Millones de mujeres se desplazan de unos países a otros para cuidar niños y adultos mayores y esto tiene unos efectos todavía hoy desconocidos en toda su dimensión. Los países de donde proceden (Bolivia, Ecuador, Paraguay, Filipinas o México) padecen las consecuencias de las migraciones de mujeres, el deterioro de los cuidados en su propia población (el drenaje de cuidados) y se construyen familias transnacionales con grandes costos de relación y emocionales.

\section{Cuidados y reproducción social}

El cuidado es una parte integral del sistema de reproducción social. Las personas nacen y son extremadamente vulnerables en la etapa de crianza y necesitan protección para satisfacer sus necesidades cotidianas (alimento, vivienda, vestido, higiene, educación). El mantenimiento de la vida requiere de un autocuidado permanente, o de alguien que se ocupe de los demás, cosa que tradicionalmente han hecho las mujeres. Además, las personas enferman o se lesionan, envejecen y pueden llegar a situaciones de dependencia que les impidan la autonomía personal. Cuidar es atender estas necesidades de la vida diaria, que se modifican en las etapas de la vida, y es imprescindible para la reproducción de los seres humanos.

Quién necesita cuidados y en qué consisten es algo construido socialmente y varía grandemente entre unas sociedades y otras. De modo similar, el cuidado es provisto en una variedad de formas institucionales, lo que incluye los servicios públicos y los servicios del mercado, la familia y la comunidad. Es lo que Mary Daly y Jane Lewis (2011) han denominado "cuidado social" y lo que Sarah Razavi (2007) caracteriza como "diamante del cuidado" (familia, mercado, Estado y comunidad).

Las normas sociales hacen que unas formas de cuidado sean más aceptables que otras, pero las decisiones políticas impactan en el tamaño de cada uno de los puntos del diamante del cuidado, y también en la calidad de esta actividad. Envejecer en el hogar, por ejemplo, es la aspiración culturalmente mayoritaria en España, por ejemplo, y como las políticas públicas son débiles, las familias utilizan un complejo "mosaico de recursos de cuidado": cuidado directo, ayuda familiar, ayuda comunitaria, utilización 
de servicios de cuidado públicos y privados, empleadas en el hogar. Y quiero añadir aquí un elemento que me parece esencial, y es tener en cuenta el sentir y las necesidades de las personas que necesitan cuidados o que requieren de apoyos para tener autonomía propia: deben tener derecho a decidir dónde quieren vivir, con quién, si tienen opciones para trabajar y dónde.

\section{La democratización de los cuidados}

Los cuidados están desigualmente repartidos. Recaen desproporcionadamente en las familias; recaen desproporcionadamente en las mujeres; y generan nuevas desigualdades basadas en componentes de clase y étnicos. Hay que avanzar hacia el derecho de ser cuidado y de cuidar en un sistema de cuidados sostenible y renovador (es decir, que no reproduzca las injusticias de género y las injusticias sociales): hay que democratizar los cuidados.

El término democratización de los cuidados es potente y evocador. Supone plantear una organización social del cuidado basada en valores democráticos tanto para las personas que los reciben como para quienes los proporcionan. Surge de una propuesta de Sandra Ezquerra y Elba Mansilla (2018) que asume el ayuntamiento de Barcelona como guía de la intervención social en este tema y que hemos empezado a utilizar en el marco académico como referencia y propuesta política. La democratización de los cuidados pasa por los siguientes ejes (que representamos en el Gráfico 1):

"Promover el reconocimiento del cuidado y de su centralidad;

"Socializar las responsabilidades del cuidado;

» Repartir el cuidado entre hombres y mujeres, para eliminar la (mal)división sexual del trabajo;

"Tener en cuenta los derechos y las demandas de las personas receptoras de cuidados, en función de su ciudadanía y no solo como consumidores.

Cada una de estas dimensiones es un paso positivo hacia la democratización de los cuidados, pero el avance de solo una de ellas constituye una democratización parcial o incluso un retroceso. Por ejemplo, si no se dan de forma simultánea una socialización del cuidado y un reconocimiento social de este, esta socialización podría darse en

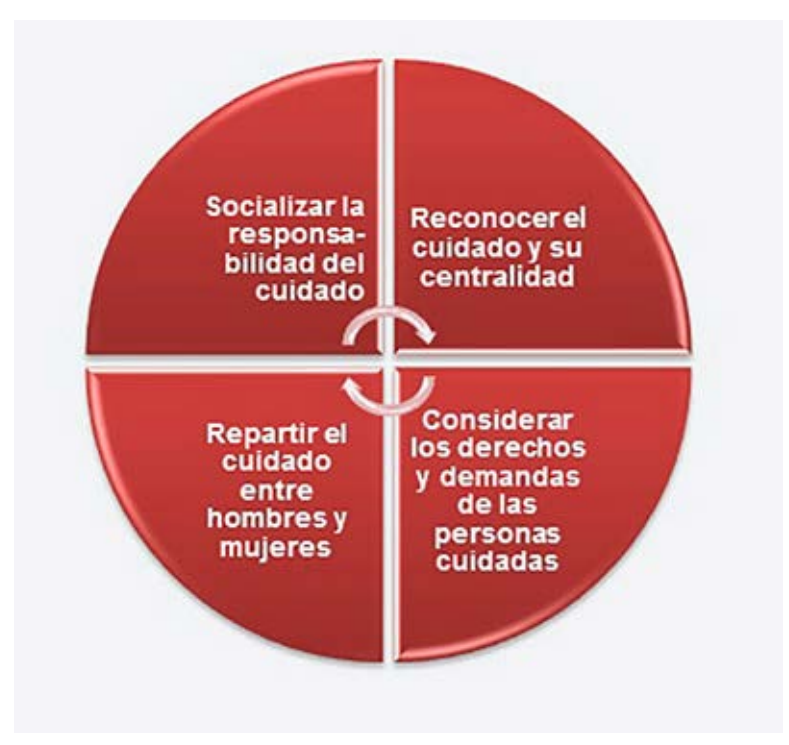

Gráfico 1. La democratización de los cuidados 
una dirección mercantilizadora y perjudicar el derecho al cuidado de los colectivos pobres. Por esto, en el gráfico, las cuatro dimensiones aparecen relacionadas. Veamos el contenido de cada una de ellas.

\section{Reconocimiento del cuidado y de su centralidad}

Los cuidados han sido especialmente invisibles por tres razones básicas. Porque es la familia la principal institución en que se llevan a cabo, de forma gratuita y altruista; porque es difícil catalogarlos como formas de trabajo, al estar impregnados de afecto y obligación moral; y finalmente, porque son las mujeres quienes los llevan a cabo y están fuertemente naturalizados. Estos factores dificultan la posibilidad de percibir su valor económico, así como su carácter crucial para la reproducción social.

Según la Plataforma de Acción de Beijing, reconocer el cuidado significa visibilizar su naturaleza, sus dimensiones y el papel que juega en contextos específicos. Este reconocimiento del cuidado se consigue por medio de su valoración simbólica y social, la garantía de un acceso universal a los cuidados desde la singularidad y la garantía de que el cuidado no se realiza a costa de los derechos de otras personas.

»Valoración simbólica y social. Se trata de eliminar el menosprecio o la trivialización del cuidado en tanto se considera una actividad de mujeres. Hay que descentrar las normas sociales masculinistas que invisibilizan la importancia del trabajo de cuidados. Hay distintas iniciativas que pueden promover esta valoración simbólica y social de estas tareas: dar visibilidad a grupos de crianza, o a las iniciativas comunitarias de adultos mayores (cohousing), y también promover las nuevas masculinidades para informar, orientar y acompañar a los hombres en momentos de transición del ciclo vital y poder generar contextos favorables para que puedan asumir y responsabilizarse de su rol de cuidadores.

» Garantía de acceso universal desde la singularidad. Universalidad y singularidad son dos criterios esenciales para analizar y promover la democratización del cuidado y establecer su centralidad social. La universalidad supone que todas las personas que necesitan cuidados y apoyo puedan obtenerlos. La singularidad implica que la provisión del cuidado tenga en cuenta las necesidades y circunstancias específicas de las personas y familias demandantes.

» No se realiza a costa de otras personas. Ello supone 1) comprender que las personas cuidadoras en el ámbito familiar sufren situaciones de aislamiento y fuertes tensiones; 2) comprender que las personas que necesitan cuidados tienen sus preferencias, lógicas, derechos y agencia; 3 ) dignificar los empleos de cuidados. Son trabajos de escaso prestigio porque los ejercen mayoritariamente mujeres y esto es así porque se perciben como una prolongación de los que las mujeres hacen en el hogar. Por esto, no se exige formación y están escasamente profesionalizados, los salarios son más bajos que en otros sectores y hay fuerte rotación laboral. Además de incidir en la calidad del servicio, dignificar este tipo de empleos resulta necesario para retener a las mujeres en ellos y para animar a los hombres a que entren en el sector.

\section{Socialización de las responsabilidades de cuidado}

Socializar el cuidado significa examinar hasta qué punto se desplaza del ámbito familiar hacia otros espacios y actores, como la comunidad, las administraciones públicas o el mercado. El cuidado es una piedra angular en la provisión de bienestar, y es en este punto que se plantea cuál es el papel que se ha de otorgar a la familia, al Estado, al mercado y a la comunidad en la organización social del cuidado. 
Las políticas sociales son imprescindibles para hacer efectivo el derecho a ser cuidado y a cuidar. Varían entre unos países y otros y otros tienen en común el proporcionar tiempo (las políticas de conciliación, permisos y excedencias), prestaciones económicas (directas o mediante la fiscalidad) y servicios (escuelas infantiles, asistencia a domicilio, centros de día, residencias, etc.). Para un sistema de cuidados sostenible, hay que socializar el cuidado, no mantenerlo solo en la familia ni atribuirlo únicamente a las mujeres. La provisión pública de cuidados es una medida redistributiva en términos de justicia social y de género, que garantiza el acceso universal con independencia de los ingresos individuales.

El mercado es otro de los agentes en la provisión de servicios de cuidado, pero este término esconde una gran variabilidad de situaciones: empleadas en el hogar a partir de la contratación directa, el sector lucrativo del mercado, y las empresas no lucrativas. Los servicios de mercado lucrativos pueden ser útiles para una parte de la población, aunque sea pequeña, pero el sistema no puede sustentarse en ellos porque generan inequidad. En cambio, sí puede potenciarse la colaboración público-privada con entidades o asociaciones que conocen en profundidad la situación de determinados colectivos sociales. Y también hay que promover que el empleo doméstico tenga condiciones de trabajo dignas, como las ha de tener cualquier empleo.

$\mathrm{Ni}$ todo ha de proceder de la familia ni del Estado protector y, en este sentido, la organización de la sociedad civil en formas de autogestión o comunitarias constituye un marco apropiado para la provisión de cuidados y un factor también de innovación social. Parte de la crisis de los cuidados se relaciona con el debilitamiento de los vínculos de parentesco y comunitarios, pero se constata también cómo estos vínculos se reactivan en situaciones de necesidad y el importante papel que en estos casos ejerce la comunidad. Actualmente, están surgiendo nuevas formas de redes de cuidados en contextos educativos, vecinales o locales, o entidades que con ayuda pública hacen posible incorporar voluntariado a las actividades de cuidado (comedores infantiles, grupos de crianza, viviendas compartidas — cohousing —, grupos de ayuda mutua, bancos del tiempo). Vale la pena impulsar las iniciativas comunitarias. En España se cometió un gran error al respecto: el impulso de las políticas públicas mató muchas organizaciones comunitarias. No tiene por qué ser así. Me consta que en Argentina hay iniciativas interesantes, tal como muestran los trabajos de Marisa Fournier (2016) sobre comedores comunitarios en los barrios periféricos de Buenos Aires, o las experiencias que describe Eleonor Faur (2014) sobre los jardines comunitarios, conveniados entre la administración pública y organizaciones de la sociedad civil. Son iniciativas que son hoy referencia en otros contextos, y vale la pena no dejarlas morir. Se puede impulsar en este sentido la colaboración público-comunitaria y, desde el sistema público, brindar apoyo técnico o incluso material a iniciativas de la ciudadanía.

\section{Repartir el cuidado entre hombres y mujeres}

La propuesta de Nancy Fraser (2008) del modelo de 'paridad en el cuidado' va en la línea de implicar a hombres y mujeres por igual en la obtención de ingresos para vivir y en las prácticas de cuidado. Se trataría pues de que hombres y mujeres, de forma equivalente, tuvieran una vida integrada por el hecho de ganar un salario, la práctica del cuidado, la implicación en la vida asociativa, que deje tiempo libre para el ocio. Es una propuesta que se sitúa en el terreno de lo utópico o de lo deseable, y Fraser no concreta cómo se llega a alcanzar, pero indica que si hoy no actuamos guiándonos por esta concepción, no hay posibilidad alguna de que nos acerquemos a ella algún día.

El impulso de nuevas masculinidades que integren el cuidado como un aspecto vital es una medida importante, pero ha de ir acompañada de otras iniciativas. 
Destacamos la necesidad de promover una conciliación corresponsable entre hombres y mujeres desde el inicio del ciclo vital. En este sentido, conseguir permisos de paternidad y maternidad iguales e intransferibles, siguiendo la tendencia iniciada en algunos países europeos, es un objetivo que tiene como triple ventaja implicar a los hombres en las tareas de crianza, eliminar los prejuicios laborales respecto de las mujeres y situar la reproducción de la vida como un aspecto de importancia social y no solo individual o de pareja.

Pero los hombres se han de implicar también en el cuidado de personas adultas en situación de dependencia, y este se diferencia del cuidado de la infancia en motivación y compromisos. Mientras que el cuidado de la infancia tiene para los hombres el estímulo de la modernidad y se asocia a los ideales de igualdad entre hombres y mujeres, no sucede lo mismo con el cuidado de personas adultas, que tiene, además, una percepción social negativa. Uno de los retos más importantes en estos momentos es justamente la implicación de los hombres en el cuidado de adultos mayores, ya sea porque lo hagan de forma sobrevenida, ya sea porque constituyen un nuevo agente a incorporar en el trabajo familiar y comunitario (Comas d'Argemir, 2016). Hay que reconocer las necesidades específicas de los hombres implicados en los cuidados de larga duración, pues no tienen referentes en los que apoyarse.

Es importante, por otro lado, que los hombres se incorporen a los trabajos remunerados de cuidados. Ya hemos comentado la necesidad de dignificar este tipo de empleos. La escasa valoración y bajos salarios de los empleos de esta categoría desaniman a los hombres a introducirse en el sector y contribuyen a incrementar la brecha de género. Paradójicamente, la incorporación de más hombres en este ámbito laboral le podría otorgar más reconocimiento y prestigio (Himmelweit y Land, 2011).

\section{Tener en cuenta las demandas y derechos de las personas receptoras de cuidado y apoyo, en función de su ciudadanía y no solo como consumidores, clientes o usuarios}

¿Quiénes son las personas receptoras de cuidado? Todos nosotros. El autocuidado es una situación común: nos cuidamos y cuidamos a las personas de nuestro alrededor. Todas las personas necesitamos cuidados a lo largo de nuestra vida, pero en determinadas etapas y situaciones se requiere que unas personas cuiden de otras: la infancia, la vejez frágil, las personas con enfermedades crónicas o impedimentos funcionales que les impiden tener autonomía (discapacidades). A estos colectivos se dirigen específicamente las políticas de cuidado y apoyo, aunque esto no ha de hacernos olvidar que el cuidado es algo mucho más amplio, que se prodiga en el día a día. Tampoco ha de hacernos olvidar que todos los seres humanos somos vulnerables.

La democratización de los cuidados implica tener en cuenta las demandas y derechos de las personas que reciben cuidados y apoyo. Un ejemplo de demandas de derechos, con una crítica profunda a la organización del sistema actual, lo proporcionan las personas con discapacidad, que rechazan ser institucionalizadas, denuncian el carácter jerárquico, paternalista y burocrático de las políticas sociales, y reclaman su capacidad de agencia y de independencia.

Recuerdo que en España, cuando empezaron a conocerse los primeros borradores de la denominada Ley de Dependencia, las personas con discapacidad se pusieron en alerta (Comas d'Argemir, 2015). Las principales organizaciones de discapacitados se movilizaron tanto en su dimensión reivindicativa como en su capacidad para proveer servicios. Considero especialmente interesante el discurso que el Foro de Vida Independiente introdujo en el debate, pues representa un nuevo paradigma. Reivindican que su situación no debe ser confundida con la de las personas dependientes. Por el 
contrario, afirman que las personas con discapacidad devienen dependientes si no cuentan con las ayudas técnicas y sociales que faciliten su independencia. Por esto prefieren hablar de diversidad funcional y no de discapacidad. No se resignan a ser institucionalizados ni a acogerse a medidas concebidas para proteger personas con pocas opciones vitales, porque resultan claramente insuficientes para las aspiraciones de quienes reivindican la normalización de su situación en la sociedad y quieren ser tratados como ciudadanos y no como enfermos. Su principal reivindicación es poder tener un asistente personal, que es quien ha de ayudar a realizar las actividades básicas de la vida diaria, acceder a los medios de transporte, al trabajo y a la educación, así como a todas las actividades de participación social. Permite, en definitiva, llevar una vida independiente en un hogar propio.

El caso de los adultos mayores era distinto. De hecho, la mencionada Ley de Dependencia estaba pensada básicamente para ellos. Pretendía desmedicalizar la fragilidad asociada a la vejez e impulsar el derecho a ser cuidado desde los servicios de carácter social. La normativa daba como prioridad crear servicios públicos de cuidado, especialmente los de atención domiciliaria, pero también centros de día, residencias asistidas y sociosanitarios, y concebía las prestaciones económicas como excepcionales. En la práctica, sin embargo, las prioridades se invirtieron, y las prestaciones económicas superaron a los servicios, en parte porque las necesidades acumuladas hacían más viable dar dinero que crear servicios, y en parte porque envejecer en el hogar es un ideal culturalmente muy arraigado y se prefería la prestación. Me refiero específicamente a ello porque contribuyó a crear un sistema frágil, y cuando se aplicaron las políticas de austeridad a raíz de la crisis económica fue muy fácil eliminar las prestaciones económicas mediante los recortes presupuestarios.

Respecto de la infancia, ha costado entender que los niños y niñas tienen derechos por sí mismos y no solo como "menores" sujetos a intervención, protección y vigilancia. Cuesta también implementar políticas públicas desde la perspectiva infantil, atendiendo a sus deseos y opiniones. ¿Cómo trabajar con la infancia y no sobre la infancia? ¿Cómo tratarlos desde una perspectiva ciudadana y no solo en su condición de menores, que los inferioriza? Es necesario abordar una verdadera participación de la infancia, en su calidad de ciudadanos, y que no sean solo las personas adultas quienes decidan sobre sus vidas porque se los considere seres vulnerables o inacabados, y aunque no voten. Se trata de dar voz a la infancia, de capturar sus preocupaciones y aspiraciones antes de que sean interpretadas desde una perspectiva adulta. Se trata también de reconocer sus experiencias y singularidades, pues la infancia no es una categoría homogénea. Seguro que todos nosotros tenemos ejemplos del sentido común que preside la manera de ver la realidad y las opiniones de niños y niñas.

\section{De la obligación al compromiso}

Afrontar las nuevas y crecientes necesidades de cuidados es un reto para las sociedades actuales y, tal como hemos intentado mostrar, las repercusiones tienen un alcance global, afectan a las familias, interpelan a los poderes públicos y contribuyen a que crezcan los servicios de mercado. El derecho a cuidar y a ser cuidado requiere poner en juego los recursos de las familias, pero también de los poderes públicos y de la comunidad. Requiere también organizar el sistema de cuidados desde las premisas de la justicia social y de la justicia de género para conformar un sistema sostenible. Hemos propuesto una democratización de los cuidados basada en la socialización de las responsabilidades de cuidado, en su reconocimiento y centralidad, en el reparto entre hombres y mujeres y en la consideración de las demandas de las personas cuidadas. 
La ética del cuidado, por otro lado, ha de ser fruto del compromiso y no de la obligación. Pasar de la obligación de cuidar al compromiso de cuidar implica una transformación social importante. El compromiso, como nos recuerda Margaret Mead (1980), es fruto de dar prioridad a unas opciones frente a otras. Mientras el cuidado sea provisto por medio de las obligaciones familiares de forma no pagada y no libre y se asigne a solo las mujeres, la igualdad de género no se conseguirá, tampoco la justicia social, ni será posible construir un sistema de cuidados sustentable. El compromiso es necesario, tanto a nivel individual como social. Necesitamos una agenda política que considere los cuidados como un aspecto central del funcionamiento de las sociedades, que sea sensible a los conflictos de intereses en la familia y que reparta los cuidados de forma más equitativa. El derecho a cuidar y a ser cuidado se sitúa así en términos de compromiso y no de obligación (compromiso de las familias, pero también de los poderes públicos y del conjunto de la sociedad); en términos de moralidad y no de materialidad.

\section{Financiamiento:}

Este documento es resultado del financiamiento del Ministerio de Economía, Industria y Competitividad. FEM2017-83517-R. España. "El compromiso de los hombres con los cuidados de larga duración. Género, generaciones y culturas del cuidado".

\section{Agradecimientos:}

Agradezco el financiamiento obtenido por parte del Ministerio de Economía y Competitividad del Gobierno de España. También agradezco la colaboración y entrega del equipo de investigación que compone el proyecto citado.

\section{Sobre la autora}

Dolors Comas-d'Argemir es doctora y catedrática de Antropología Social y Cultural de la Universidad Rovira i Virgili. Es coordinadora del programa de doctorado de Antropología y Comunicación. 


\section{Q Bibliografía}

» Bhattacharya, T. (2013). Explaining gender violence in the neoliberal era. Internacional Socialist Review, 91. Recuperado de https://isreview.org/issue/91/explaining-gender-violence-neoliberal-era

" Boris, E. y Parreñas, R. (Ed.) (2010). Intimate labors: Cultures, technologies and politics of care. Standford: Stanford University Press.

» Carrasco, C., Borderías, C. y Torns, T. (Eds.) (2011). El trabajo de cuidados. Historia, teoría y políticas. Madrid: La Catarata.

»Carsten, J. (2004). After kinship. Cambridge: Cambridge University Press.

"Comas d’Argemir, D. (1998). Antropología económica. Barcelona: Ariel.

"Comas d'Argemir, D. (2015). Los cuidados de larga duración y el cuarto pilar del Estado del bienestar. Revista de Antropología Social, 24, 375-404. doi: 10.5209/rev_RASO.2015. v24.50663

"Comas d'Argemir, D. (2016). Hombres cuidadores: barreras de género y modelos emergentes. Psicoperspectivas. Individuo y Sociedad, 15(3), 10-22. doi: 10527/PSICOPERSPECTIVAS-VOL15-ISSUE3-FULL-750

"Comas d'Argemir, D. (2017). El don y la reciprocidad tienen género: las bases morales de los cuidados. Quaderns-e de l'Institut Català d'Antropologia, 22(2), 17-32. Recuperado de http://www.antropologia.cat/files/2_ComasdArgemir.pdf

" Comas d'Argemir, D. y Roigè, X. (2018). Introduction. Between family and State. The new faces of ageing in Europe. Ethnologie française, 171, 389-400. Recuperado de https://www. cairn-int.info/article-E_ETHN_183_0389--between-family-and-state-thenew-faces.htm

"Comas d'Argemir, D. y Soronellas, M. (2019). Men as Carers in Long-Term Caring. Doing Gender and Doing Kinship. Journal of Family Issues, 40(3), 315-339. doi: 10.1177/0192513X18813185

"Constable, N. (2009). The commodification of intimacy: Marriage, sex, and reproductive labor. Annual Review of Anthropology, 38, 49-64. doi:10.1146/annurev. anthro.37.081407.085133

》Coraggio, J.L. (1999). Política social y economía del trabajo. Alternativas a la política neoliberal para la ciudad. Madrid: Miño y Dávila.

"Daly, M. y Lewis, J. (2011). El concepto de 'social care' y el análisis de los Estados del Bienestar contemporáneos. En C. Carrasco, C. Borderías y T. Torns (Eds.), El trabajo de cuidados; historia, teoría y políticas (pp. 225-251). Madrid: La Catarata.

»Drotbohm, H. y Alber, E. (2015). Introduction. En E. Alber y H. Drotbhom (Eds.), Anthropological perspectives of care, work, kinship, and the life-course (pp. 1-19). Nueva York: Palgrave MacMillan.

» Ehrenreich, B. y Hochschild, A.R. (Eds.) (2003). Global Woman. Nannies, Maids and Sex Workers in the New Economy. Nueva York: Henry Holt and Co.

»Esquivel, V. (2011). La economía del cuidado en América Latina: poniendo a los cuidados en el centro de la agenda. El Salvador: PNUD. 
"Esquivel, V., Faur, E. y Jelín, E. (Coords.) (2012). Las lógicas del cuidado infantil. Entre las familias, el Estado y el mercado. Buenos Aires: IDES-Unicef-Unpfa. Recuperado de http://cddhh.ides.org.ar/files/2012/06/Las-1\%C3\%B3gicas-del-cuidado-infantil.-Entrelas-familias-el-Estado-y-el-mercado.1.pdf

»Ezquerra, S. y Mansilla, E. (2018). Economia de les cures i política municipal: cap a una democratització de la cura a la ciutat de Barcelona. Barcelona: Ajuntament de Barcelona, Universitat de Vic, La Ciutat Invisible Coop. Recuperado de http://ajuntament.barcelona.cat/tempsicures/sites/default/files/economia_i_politica_13.pdf

» Faur, E. (2014). El cuidado infantil en el siglo XXI. Mujeres malabaristas en una sociedad desigual. Buenos Aires: Siglo XXI.

» Finch, J. y Groves, D. (Eds.) (1983). A labour of love: women, work and caring. Londres: Routledge-Kegan.

» Fournier, M. (2016). La labor de las trabajadoras comunitarias de cuidado infantil en el conurbano bonaerense. ¿Una forma de subsidio de "abajo hacia arriba"? Trabajo y Sociedad, 28: 83-108. Recuperado de https://www.redalyc.org/articulo.oa?id=387349334005

»Fraser, N. (2008). Escalas de justicia. Barcelona: Herder.

» Gilligan, C. (2013). La ética del cuidado. Barcelona: Fundació Víctor Grífols i Lucas.

» Godelier, M. (2017). Transitions et subordinations au capitalisme. París: Éditions de la Maison des sciences de l'homme.

" Harris, O. y Young, K. (1981). Engendered structures: some problems in the analysis of reproduction. En J. S. Kahn y J. R. Llobera (Eds.), The Anthropology of Pre-capitalist Societies (pp. 107-147). Londres: MacMillan.

» Himmelweit, S. y Land, H. (2011). Reducing gender inequalities to create a sustainable care system. Kurswechsel, 4: 49-63.

" Katz, C. (2001). Vagabond Capitalism and the Necessity of Social Reproduction. Antipode, 33(4), 709-728. doi: 10.1111/1467-8330.00207

» Mead, M. (1980). Cultura y compromiso. Estudio sobre la ruptura generacional. Barcelona: Gedisa.

" Offenhenden, M. (2017). 'Si hay que romperse una, se rompe'. El trabajo del hogar y la reproducción social estratificada (tesis de doctorado). Universitat Rovira i Virgili, Tarragona, España. Recuperado de https://www.tdx.cat/bitstream/handle/10803/460763/TESI. pdf?sequence $=1$

» Polanyi, K. (1989). La gran transformación. Crítica del liberalismo económico. Madrid: La Piqueta.

"Presta, S. y Landaburu, L. (2014). Economía social y solidaria: paradojas de la "moneda social”. Idéias, 5(2), 141-159. Recuperado de https://periodicos.sbu.unicamp.br/ojs/ index.php/ideias/article/view/8649433

" Razavi, S. (2007). The Political and Social Economy of Care in a Development Context. Conceptual Issues, Research Questions and Policy Options. Gender and Development Programme, Paper numer 3. United Nations Institute for Social Development.

» Razavi, S. y Staab, S. (2010). Underpaid and overworked: A cross-national perspective on care workers. International Labour Review, 149(4), 407-422. doi: 10.1111/j.1564913X.2010.00095.X

» Thelen, T. (2015). Care as social organization: Creating, maintaining and dissolving significant relations. Anthropological Theory, 15, 497-515. doi: 10.1177/146349961560o893 
» Tobío, C., Agulló, M. S., Gómez, M. V. y Martín, M.T. (2011). El cuidado de las personas. Un reto para el siglo XXI. Barcelona: Fundació La Caixa. Recuperado de https://multimedia.caixabank.es/lacaixa/ondemand/obrasocial/pdf/estudiossociales/vol28_completo_es.pdf

»Wallerstein, I. (1988). El capitalismo histórico. Madrid: Siglo XXI.

»Wikander, U. (2016). De criada a empleada. Poder, sexo y división del trabajo (1789-1950). Madrid: Siglo XXI. 
